\title{
Indicadores de qualidade em terapia nutricional enteral em uma unidade de terapia intensiva no interior de Rondônia
}

\author{
Quality indicators in enteral nutritional therapy in an intensive therapy unit in Rondônia
}

Indicadores de calidad en terapia nutricional enteral en una unidad de terapia intensiva en Rondônia

Priscila Nayara Vasconcelos Posso ${ }^{1 *}$, Patrícia de Souza Chagas ${ }^{1}$, Andressa Magali kopper ${ }^{1}$, Rittiela Rocha da Silva ${ }^{1}$, Aline Brito Lira Cavalcante ${ }^{1}$, Andreska Lara Silva Bonfá1, Briany Soares Rodrigues $^{1}$, Emanuelle Nogueira Negreiros ${ }^{1}$, Emilly Karine Ventura Lima ${ }^{1}$, Angela Antunes de Morais Lima'.

\section{RESUMO}

Objetivo: Aplicar e avaliar os Indicadores de Qualidade Terapia Nutricional em pacientes internados nas UTIs Adulto de um Hospital público no interior de Rondônia. Métodos: Estudo de caráter transversal, descritiva, com abordagem quantitativa por meio de dados obtidos em 2019, colhidos junto a fichas de anotações de enfermagem e do setor de nutrição. A amostra foi composta por pacientes de ambos os sexos com idade superior a 18 anos que receberam terapia nutricional enteral (TNE) no período. Resultados: Foram analisados 50 pacientes em uso de TNE exclusivo, com média de idade de $53 \pm 19,49$ e $59 \pm 20,73$ anos para homens e mulheres, respectivamente. No período analisado, foi observado que a média de volume prescrito e infundido foi de $1040,02 \pm 115,45 \mathrm{ml} / \mathrm{dia}$ e $879,31 \pm 134,51 \mathrm{ml} / \mathrm{dia}$, respectivamente. A média de calorias

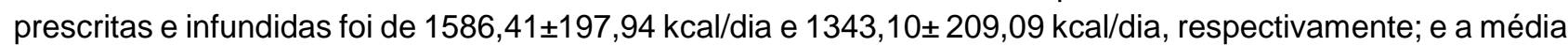
de prescrito e infundido de proteínas foi de $86,17 \pm 10,16 \mathrm{~g} /$ dia e $78,4 \pm 10,76 \mathrm{~g} / \mathrm{dia}$, respectivamente. A adequação calórica para o grupo de estudo foi de $82,3 \% \pm 23,6 \%$ e a adequação proteica de $87,5 \% \pm 11,1 \%$. Conclusão: Os IQTN aplicados estavam de acordo com as metas pré-estabelecidas. Sendo que, ainda são necessários mais treinamentos e conscientização da equipe quanto à TNE.

Palavras chave: Indicadores de qualidade em assistência à saúde, Terapia nutricional, Unidade de terapia intensiva.

\begin{abstract}
Objective: To apply and measure the Quality Indicators in Nutrition Therapy on adults patients in hospital ICUs of a public hospital in the countryside of Rondônia. Methods: This is a cross-sectional, descriptive study with a quantitative approach based on data from 2019, collected from nursing and nutrition notes. The sample consisted of patients of both sexes aged over 18 years who received enteral nutritional therapy (ENT) during the period. Results: We analyzed 50 patients that were using exclusively ENT, with an average age of $53 \pm 19.49$ and $59 \pm 20.73$ years old for men and women, respectively. It was observed that the average prescribed and infused volume was $1040.02 \pm 115.45 \mathrm{ml} /$ day and $879.31 \pm 134.51 \mathrm{ml} /$ day, respectively. The average of prescribed and infused calories was $1586.41 \pm 197.94 \mathrm{kcal} /$ day and $1343.10 \pm 209.09 \mathrm{kcal} /$ day, respectively; and the average of prescribed and infused proteins was $86.17 \pm 10.16 \mathrm{~g} /$ day and $78.4 \pm 10.76$ $\mathrm{g} /$ day, respectively. Caloric adequacy for the study group was $82.3 \% \pm 23.6 \%$ and protein adequacy $87.5 \%$ $\pm 11.1 \%$. Conclusion: The indicators applied were in accordance with the pre-established goals. Since, more training and awareness of the team regarding ENT are still needed.
\end{abstract}

Keywords: Quality indicators health care, Nutrition therapy, Intensive care unit.

${ }^{1}$ Hospital Regional de Cacoal (HRC), Cacoal - RO. *E-mail: priiposso@gmail.com 


\section{RESUMEN}

Objetivo: Aplicar y evaluar IQTN en pacientes ingresados en la Unidad de Cuidados Intensivos para Adultos (UCl) de un hospital público en el interior de Rondônia. Métodos: Este es un estudio descriptivo transversal con un enfoque cuantitativobasado en datosobtenidos en 2019, recopilados de notas del sector de enfermería y nutrición. La muestraconsistió en pacientes de ambos sexos mayores de 18 años que recibieron terapia nutricional enteral (ENT) en el período. Resultados: se analizaron cincuenta pacientes que usaban ENT exclusivo, con una edad media de $53 \pm 19.49$ y 59 años. \pm 20.73 años para hombres y mujeres, respectivamente. Durante el período analizado, se observó que el volumen pro medio prescrito e infundido era $1040.02 \pm 115.45 \mathrm{ml} /$ día y $879.31 \pm 134.51 \mathrm{ml} /$ día, respectivamente. El pro medio de calorías prescritas e infundidas fue $1586.41 \pm 197.94 \mathrm{kcal} /$ día y $1343.10 \pm 209.09 \mathrm{kcal} /$ día, respectivamente; y el pro medio de proteína prescrita e infundida fue $86.17 \pm 10.16 \mathrm{~g} /$ día y $78.4 \pm 10.76 \mathrm{~g} /$ día, respectivamente. La adecuación calórica para el grupo de estudio fue del $82,3 \% \pm 23,6 \%$ y la adecuación de las proteínas del $87,5 \% \pm$ 11,1\%.Conclusión: EI IQTN aplicado estaban en línea con los objetivos preestablecidos. Desde entonces, aún se necesita más capacitación y conciencia del equipo sobre ENT.

Palabras clave: Indicadores de calidad de la atención de salud, Terapia nutricional, Unidad de cuidados intensivos.

\section{INTRODUÇÃO}

A desnutrição é definida como o estado de insuficiência resultante da deficiência de nutrientes onde a ingestão inadequada ou inabilidade em absorver e utilizar os nutrientes podendo causar alterações na funcionalidade e prejuízo ao organismo. A desnutrição está associada ao prognóstico ruim no paciente grave, é considerada consequência e não causa, porém, subestimá-la ou ignorá-la pode trazer graves consequências ao paciente. A desnutrição apresenta alta prevalência em todo o mundo, no Brasil a desnutrição parece estar presente em $48,1 \%$ dos doentes internados. $O$ aporte nutricional adequado pela nutrição enteral (NE) é considerado uma estratégia terapêutica importante, pois diminui complicações e o tempo de permanência na unidade de terapia intensiva (UTI), minimizando custos hospitalares (FREITAS TEC, et al., 2018; RIBEIRO LMK, 2014).

O processo de desnutrição do paciente em condição crítica é rápido e devastador. O grau de inflamação e alteração metabólica desencadeada pela doença de base é responsável pela intensa perda de peso, à custa, principalmente, de massa muscular. Encarar a desnutrição do paciente grave e tratá-la com a mesma seriedade com que se tratam outras alterações a que ele é exposto é fundamental para obtenção de bons resultados, principalmente funcionais (TOLEDO DO e CASTRO MG, 2019).

A Terapia nutricional (TN) pode ser estabelecido como um conjunto de possibilidades terapêuticas empregadas para a recuperação ou manutenção do estado nutricional por meio da nutrição seja ela por via enteral e/ou parenteral, podendo além de corrigir a desnutrição, também diminuir estresse metabólico, contribuir para a redução de complicações infecciosas e clínicas. Entretanto, a terapia nutricional deve ser monitorada e avaliada continuamente devido a viabilidade de complicações (SÁ JSM e MARSHALL NG, 2015).

A terapia nutricional (TN) tem um impacto significativo na evolução clínica de um paciente hospitalizado, principalmente em pacientes internados em unidade de terapia intensiva (UTI), que apresentem anorexia e incapacidade de se alimentar por via oral por dias e até mesmo por meses, fazendo com que haja indicação para terapia nutricional enteral (TNE) (PIOVACARI SMF, et al., 2017).

$\mathrm{Na}$ unidade de terapia intensiva (UTI) pacientes críticos apresentam um intenso estresse metabólico, com acelerado catabolismo protéico e, como consequência, evoluem com depleção muscular, prejuízo do estado nutricional, com desnutrição, dentre outras alterações. O conhecimento e controle desses fatores pela equipe multiprofissional faz com que medidas possam ser adotadas com a finalidade de otimizar a utilização da terapia nutricional enteral (TNE), possibilitando o fornecimento dos nutrientes aos pacientes críticos de forma mais adequada (MENDONÇA MR, 2018; FUJINO V, NOGUEIRA LABNS, 2007). 
Os indicadores de qualidade em terapia nutricional (IQTN) são ferramentas de avaliação e monitoramento da qualidade da TN, ao identificarem possíveis dificuldades e falhas relacionadas aos protocolos de cuidados nutricionais providos ao paciente. Aplicar indicadores de qualidade pode-se garantir a eficiência nas rotinas diárias, reduzir custos, melhorar a capacidade de análise de processos e, principalmente, resultar em melhora clínica e de qualidade de vida para esses indivíduos (TOLEDO DO e CASTRO MG, 2019; SOUZA e MEZZOMO, 2016). A utilização de indicadores de qualidade é uma das formas de elevar o monitoramento da terapia nutricional enteral para padrões de qualidade exigidos atualmente nas UTI (TOLEDODO e CASTRO MG, 2015). Também podem ser utilizados para introduzir rotinas de serviço, para avaliar a adequada relação entre profissional e paciente para compor a análise crítica de aspecto nutricional da terapia, confrontando recomendações de diretrizes com a prática clínica dentro de um hospital (TOLEDO DO e CASTRO MG, 2019).

Nesse contexto, o objetivo deste estudo foi aplicar e avaliar os indicadores de qualidade da Terapia Nutricional Enteral (TNE) em pacientes internados nas Unidades de Terapia Intensiva (UTI) Adulto de um Hospital público no interior de Rondônia, a fim de analisar as conformidades e adequações das unidades em relação à terapia nutricional enteral e identificar possíveis problemas relacionados à terapia nutricional visando a melhor resposta clínica e nutricional aos pacientes.

\section{MÉTODOS}

Trata-se de uma pesquisa transversal, documental, descritiva de abordagem quantitativa, composta pela amostra de 50 (cinquenta) pacientes adultos com idade igual ou superior a 18 anos, ambos os sexos, que receberam nutrição enteral (NE) exclusiva por pelo menos 24 horas, internados em UTI de um hospital público de Cacoal-RO. Houve a dispensa do Termo de Consentimento Livre Esclarecido (TCLE), pois foram coletados dados secundários em prontuários e fichas de acompanhamento relacionado à Terapia Nutricional Enteral. $O$ projeto foi aprovado pelo Comitê de Ética em Pesquisa da faculdade Faculdade Ciências Biomédicas de Cacoal - FACIMED com número de parecer: 3.007.574. A pesquisa teve início no mês de Janeiro de 2019 e foi finalizada em abril de 2019. Durante sua execução, foram coletados dados a partir das fichas de anotações de enfermagem e das fichas e planilhas de prescrição de dietas do setor de nutrição.

Todas as informações utilizadas já faziam parte da rotina de atendimento nutricional dos pacientes internados. O levantamento de dados foi realizado com a frequência de duas a três vezes por semana em dias aleatórios, com um total de 63 dias, onde foram coletados e posteriormente empregados em formulário contendo dados sobre o leito, sexo, tempo de internação, volume/calorias/proteínas (necessidade, prescrição, infusão), fórmula utilizada e infusões não administradas (contendo o(s) motivo(s), caso sejam citados nos prontuários/fichas). Os indicadores de qualidade em terapia nutricional (IQTN) aplicados foram os propostos por Waitzberg DL (2018). Os indicadores avaliados foram: I1: Frequência de Dias de Administração Adequada do Volume Prescrito X Volume Infundido em Pacientes em terapia Nutricional Enteral (TN);I2: Frequência de Dias de Administração Adequada de Energia em Pacientes em Terapia Nutricional Enteral (TNE); e I3: Frequência de Dias de Administração Adequada de Proteínas em Pacientes em Terapia Nutricional Enteral (Tabela 1).

Tabela 1 - Indicadores e metas de qualidade em terapia nutricional utilizados no estudo.

\begin{tabular}{ccc}
\hline Frequência & Fórmulas & Metas \\
\hline (I1) Dias de administração adequada do & № de pacientes com volume adequado de & \\
volume prescrito $\times$ volume infundido em & TNE $\times 100$ & $\geq 80 \%$ \\
pacientes em terapia nutricional enteral & Número de diasavaliados & \\
(12) Dias com aporte calórico adequado no & № de dias de meta calórica $\geq 25 \mathrm{kcal} / \mathrm{kg} / \mathrm{dia} e \leq$ & \\
total de dias em terapia nutricional enteral & $30 \mathrm{kca} / \mathrm{kg} / \mathrm{dia} \quad \mathrm{X} 100$ & $\geq 80 \%$ \\
& Número de diasavaliados \\
(I3) Dias com aporte protéico adequado no & $\mathrm{N}^{\circ}$ de dias de meta protéica $\geq 1,2 \mathrm{~g} / \mathrm{kg} / \mathrm{dia}$ e $\leq$ & \\
total de dias em terapia nutricional enteral & $2,0 \mathrm{~g} / \mathrm{kg} /$ dia $\quad \mathrm{X} 100$ & $\geq 80 \%$ \\
& Número de diasavaliados & \\
\hline
\end{tabular}

Legenda: $\mathrm{NE}=$ Terapia Nutricional Enteral; kcal= Quilocaloria Kg= Kilograma.

Fonte: Posso PNV, et al., 2019. Baseado: Waitzberg DL, 2018; Luz ERL e Mezzomo TR, 2015. 
Os dados foram compilados no programa Microsoft Excel® e descritos por meio de distribuição de média e desvio padrão. Para as análises estatísticas, foi utilizado o teste t de Student dentro do ambiente do $R$ Studio. Para a avaliação do problema o método do teste $t$ Student utilizado foi para duas amostras dependentes (pareadas), adotando um nível de significância $p$-valor $<0,05$. Análise estatística descritiva foi utilizada em cada variável. A análise dos resultados foi composta por meio da construção de tabelas e gráfico.

\section{RESULTADOS}

$\mathrm{Na}$ avaliação dos 50 pacientes em uso exclusivo de TNE em duas Unidades de Terapia Intensiva. A (Tabela 2) apresenta a média de idade e tempo de internação dos indivíduos avaliados. Foi verificado que a média de idade foi de $53 \pm 19,49$ e $59 \pm 20,73$ anos para homens e mulheres, nesta ordem. A população consta de 33 (66\%) homens e 27 (54\%) mulheres.

A faixa etária variou de 21 a 88 anos, estatisticamente não havendo diferença significativa entre os sexos. Quanto ao tempo de internação, a média verificada foi de $26 \pm 23,28$ e $42 \pm 30,74$ dias para homens e mulheres, respectivamente.

Tabela 2 - Médias de idade e tempo de internação em relação ao gênero, de um hospital público de Cacoal, Rondônia - 2019.

\begin{tabular}{cccccc}
\hline & \multicolumn{2}{c}{ Masculino } & \multicolumn{2}{c}{ Feminino } \\
\hline Variáveis & Média & $\mathbf{\pm D P}$ & Média & $\mathbf{\pm D P}$ & p-valor* \\
\hline Idade (anos) & 53,5 & 19,49 & 59 & 20,73 & 1,6075 \\
Tempo Internação (Dias) & 26 & 23,28 & 42 & 30,74 & 1,3860 \\
\hline
\end{tabular}

Legenda: *Teste t de Student. DP = desvio padrão. Fonte: Posso PNV, et al., 2019.

Segundo o protocolo do hospital, os pacientes devem atingir sua meta energética de $25-30 \mathrm{kcal} / \mathrm{kg} / \mathrm{dia}$ e meta proteica de $1,2 \mathrm{~g}-2,0 \mathrm{~g} \mathrm{ptn} / \mathrm{kg} / \mathrm{dia}$ no $4^{\circ}$ dia após início de terapia nutricional sendo que nesses dias de progressão de dieta são utilizadas fórmulas normocalóricas e/ou hipercalóricas, com ou sem módulo de proteína, dependendo da necessidade do paciente. De acordo com a (Tabela 3) observamos as médias dos 63 dias avaliados, onde é possível observar uma diferença significativa entre o volume prescrito e o infundido (infundido menor que o prescrito).

Tabela 3 - Médias de volume prescrito e infundido de dietas enterais, de um hospital público de Cacoal, Rondônia - 2019.

\begin{tabular}{|c|c|c|c|c|c|}
\hline & Prescrito & & Infundido & & \\
\hline Volume & Média & $\pm \mathrm{DP}$ & Média & $\pm \mathrm{DP}$ & p-valor* \\
\hline Volume (ml) (63dias) & 1040,02 & 115,45 & 879,31 & 134,51 & $<0,0001$ \\
\hline Valor Máxima/Mínima & Valor mín & Valor Máx & Valor Mín & Valor Máx & - \\
\hline Volume $(\mathrm{ml})$ (63 dias) & 589,09 & 1200,00 & 457,31 & 1170,75 & - \\
\hline
\end{tabular}

Legenda: ${ }^{\star}$ Teste t de Student. DP = desvio padrão. Fonte: Posso PNV, et al., 2019.

Quanto à necessidade calórica e proteica, comparou-se o valor infundido com a necessidade de calorias e proteínas nos 63 dias avaliados, onde era esperado que o alcance da meta de calorias $25-30 \mathrm{kcal} / \mathrm{kg} / \mathrm{dia}$ e proteínas de 1,2-2,0g ptn/kg/dia fossem atingidos.

Entretanto, houve diferença nos resultados demonstrados entre as duas variáveis (infusão e necessidade), indicando que, em média, tanto o infundido de calorias quanto as proteínas estavam abaixo das necessidades diárias prescritas aos pacientes; os dados estão apresentados na (Tabela 4). 
Tabela 4 - Médias de infusão e necessidades de calorias e proteínas, de um hospital público de Cacoal, Rondônia - 2019.

\begin{tabular}{cccccc}
\hline & \multicolumn{2}{c}{ Prescrito } & \multicolumn{2}{c}{ Infundido } \\
\hline Volume & Média & $\mathbf{\pm D P}$ & Média & $\mathbf{\pm D P}$ & p-valor $^{\star}$ \\
\hline Calorias (cal) (63 dias) & 1586,41 & 197,94 & 1343,19 & 209,09 & $<0,0001$ \\
Proteínas (g) (63 dias) & 86,17 & 10,16 & 78,45 & 10,76 & $<0,0001$ \\
\hline Valor Máxima/Mínima & Valor mín & Valor Máx & $\begin{array}{c}\text { Valor } \\
\text { Mín }\end{array}$ & Valor Máx & - \\
\hline Calorias (cal) (63 dias) & 910,90 & 1900,37 & 688,04 & 1885,00 & - \\
Proteínas (g) (63 dias) & 45,54 & 101,44 & 44,04 & 98,88 & - \\
\hline
\end{tabular}

Legenda: *Teste t de Student. DP = desvio padrão. Fonte: Posso PNV, et al., 2019.

Para a avaliação de adequação das necessidades calóricas e protéicas os resultados estão apresentados na Tabela 5, onde observamos que mesmo quando as variáveis de aporte calórico e protéico estão abaixo das necessidades diárias prescritas aos pacientes, não houve diferença estatística significante entre os valores de adequação da meta (<80\% ou $\geq 80 \%$ ) de caloria e proteína obtidos. Sendo que a adequação calórica foi de $82,3 \pm 23,6 \%$ e adequação protéica de $87,5 \pm 11,1 \%$.

Tabela 5 - Análise das necessidades calórica e proteicas dos 63 dias.

\begin{tabular}{ccccc}
\hline \multicolumn{5}{c}{ Adequação da meta } \\
\hline Variáveis & $<80 \%$ & $\geq 80 \%$ & Total & p-valor \\
\hline Caloria & $17,7 \%$ & $82,3 \%$ & $100 \%$ & 23,6 \\
Proteína & $12,5 \%$ & $87,5 \%$ & $100 \%$ & 11,1 \\
\hline
\end{tabular}

Legenda: *Teste t de Student. DP = desvio padrão. Fonte: Posso PNV, et al., 2019.

Os motivos para não administração ou interrupção da administração das dietas enterais (alimentação enteral), foram: pausa para procedimento $22,7 \%$, intercorrências gastrointestinais $15,9 \%$, suspensão por ordem médica $18,1 \%$, retirada de sonda $29,5 \%$ e outros motivos $11,3 \%$ (Figura 1 ).

Figura 1 - Motivos para não administração ou interrupção de dietas enterais, em um hospital público de Cacoal, 2019.

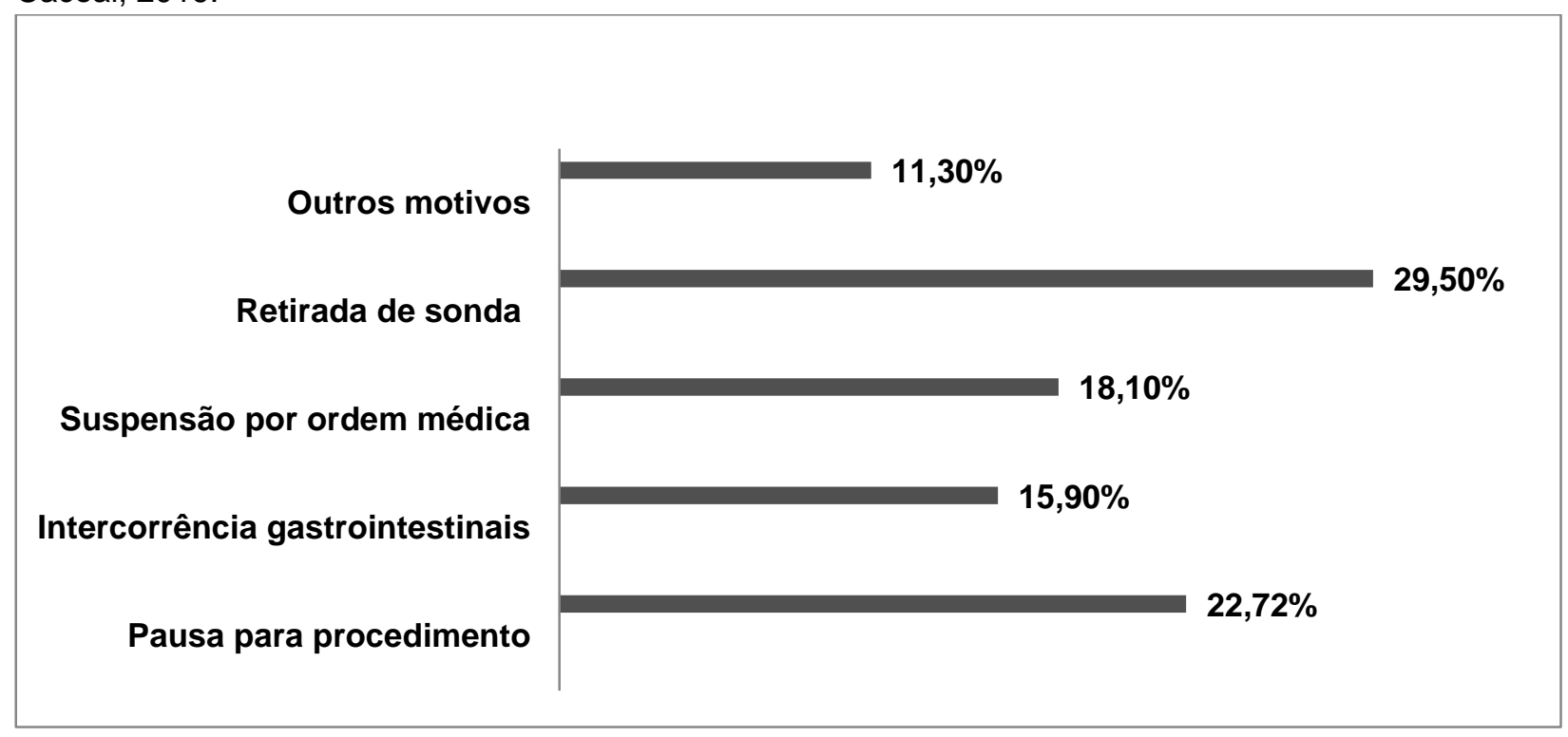

Legenda: *Outros motivos: Instabilidade hemodinâmica, parada cardíaca, atraso infusão, dieta zero.

Fonte: Posso PNV, et al., 2019. 


\section{DISCUSSÃO}

A desnutrição presente em indivíduos hospitalizados vem sendo frequentemente um fator complicador para o prognóstico clínico. A terapia nutricional é reconhecida por trazer bons resultados na terapia intensiva, as evidências sugerem que a TNE é capaz de atender à demanda dos nutrientes necessários ao paciente crítico, além de manter a integridade intestinal e reduzir o tempo de internação e risco de infecções e complicações e impacta favoravelmente o desfecho dos pacientes.

Apesar dos benefícios do uso da nutrição enteral, nem todos os pacientes admitidos na UTI recebem toda a nutrição prescrita, visto que há diversas barreiras que levam à interrupção da oferta nutricional planejada, portanto, avaliar a eficácia e a qualidade desse tratamento é fundamental (WALCZEWSKI MRM, ET AL., 2019).

Nas UTIs estudadas, foi observado que os pacientes apresentavam uma média de idade de $\geq 53$ anos, onde o grupo feminino apresentou maior média de idade em comparação ao grupo masculino. Em seu estudo transversal descritivo Silva RR, et al., (2020), realizaram coleta de dados em prontuários de uma UTI-Adulto, que recebeu 723 internações, onde 635 pacientes eram do sexo masculino $(55,7 \%)$, o que se assemelha a este estudo, onde a maioria dos indivíduos pertencia ao sexo masculino $(60 \%)$, demonstrando que pacientes do sexo masculino possivelmente são mais propensos a ignorar os problemas de saúde e por consequência demoram a buscar pelo atendimento médico após o estabelecimento da doença.

Pacientes críticos, que permanecem por mais de 48 horas na UTI, devem ser considerados pacientes em risco de desnutrição. Conhecer as características e limitações da TNE em cada local, a fim de traçar as estratégias terapêuticas mais adequadas pode reduzir a gravidade da doença, as complicações e o tempo de permanência na UTI, impactando no desfecho do paciente de forma favorável (JESUS KMG, ET AL., 2019).

No presente estudo a média de tempo de internação foi de $26 \pm 23,28$ e $42 \pm 30,74$ dias para homens e mulheres, respectivamente; sendo que o tempo de permanência da UTI foi identificado como fator associado com o aumento da mortalidade dos pacientes. Segundo Oliveira SO, et al., (2011) foram observados uma mortalidade em UTI significativamente maior nos pacientes com tempo de internação superior a 21 dias (24\%) comparado a pacientes com menor tempo de internação (11\%).

Os indicadores de qualidade na TNE e sua avalição periódica são úteis na análise da qualidade da TNE ofertada aos pacientes. Segundo os dados da Tabela 3, nota-se que o volume das infusões da dieta enteral foi menor em relação ao volume prescrito. A diferença do volume de dieta enteral infundida da prescrita pode ser justificada se o volume da dieta for alterado após uma intercorrência em pacientes gravemente enfermos e clinicamente instáveis.

Em um estudo realizado por Martins JR (2012) com 152 indivíduos adultos, que incluiu 36 pacientes em enfermaria e 116 pacientes em Unidade de Terapia Intensiva (UTI), concluiu-se que $20 \%$ dos pacientes tiveram inadequação entre prescrição e infusão de terapia nutricional enteral e que as principais causas para a não infusão de dieta enteral conforme o prescrito foi: problemas logísticos operacionais, estase gástrica e perda acidental da sonda de alimentação enteral.

Outro estudo realizado com 51 pacientes adultos observou-se que em média 70,2\% dos pacientes receberam volume inferior a $80 \%$ do volume prescrito pelo nutricionista, onde as principais intercorrências que contribuíram para baixa administração de dieta enteral foram: 18,9\% jejum para exames ou outros procedimentos e $11 \%$ problemas relacionados à bomba de infusão.

Em seu estudo McClave SA, et al., (2009) verificaram que os pacientes que receberam volume de nutrição enteral próximo de $100 \%$ evoluíram com menor taxa de complicações infecciosas, menos tempo de permanência hospitalar e com tendência a menor mortalidade.

A despeito dos resultados obtidos, percebe-se que a quantidade de calorias e de proteínas infundidas foram menores em relação à quantidade prescrita aos pacientes. Desse modo, houve diferença estatística dos mesmos em relação ao prescrito versus infundido (Tabela 4). José IB, et al., (2018) em seu estudo observaram que seus resultados da infusão de calorias e proteínas evidenciaram que a dificuldade da infusão 
de terapia nutricional enteral (TNE) em pacientes críticos ocorre independentemente da gravidade/condição do paciente.

Contudo mesmo que a infusão de volume, calorias (kcal) e proteínas $(\mathrm{g})$ foram menores que o prescrito, a adequação das necessidades calóricas e protéicas encontradas foram satisfatórias, onde a adequação de calorias foi de $82,3 \pm 23,6 \%$ e adequação protéica de $87,5 \pm 11,1 \%$; comparando-se com os estudos de Oliveira CCG e Segadilha NLAL; (2019) onde a média de calorias ofertada foi de 1511,2 $\pm 575,8 \mathrm{kcal} / \mathrm{dia}$ e de proteína, $67,9 \pm 25,4 \mathrm{~g} /$ dia, o que representa, respectivamente, uma adequação de $88 \pm 38,7 \%$ e $86,8 \pm$ $24,8 \%$. Embora os resultados sejam considerados satisfatórios, houve interrupção na infusão da dieta enteral sem descrição do motivo em alguns dos pacientes avaliados. Essa interrupção evita que o paciente atinja o volume prescrito e suas necessidades nutricionais, podendo ser um fator responsável pela dificuldade no tratamento clínico desses pacientes.

Ribeiro LMK, et al., (2014) avaliaram 93 pacientes internados em uma UTI de um hospital universitário em São Paulo, onde identificaram uma adequação de $82,2 \%$, tanto para calorias quanto para proteínas. Estudos sobre resultados bem sucedidos de pacientes criticos em uso de TNE correlacionaram baixa oferta de energia e proteína com piores resultados clínicos, evidenciando um aumento de complicações infecciosas, dias de ventilação mecânica, maior permanência na UTI e maior frequência de lesões por pressão. Segundo Weijs PJM,et al., (2012), a definição e o alcance individual de metas de energia e proteína, reduz em $50 \%$ a mortalidade de pacientes críticos.

Sabemos que a TN seja de suma importância durante o processo de recuperação e manutenção do paciente crítico, porem esta não é isenta de riscos, como por exemplo, complicações gastrointestinais ou mecânicas. Dentre os motivos relacionados à interrupção da infusão da TNE, a retirada de sonda foi a maior causa com 29,5\%, para a menor adequação das necessidades nutricionais nos pacientes avaliados, o que demonstra a falta de conscientização da equipe quanto à importância do aporte nutricional adequado aos pacientes de UTI. Segundo a literatura de Toledo DO e Castro MG (2019) dados recentes de hospitais do Brasil revelam $34 \%$ de saída de sondas enterais em pacientes sob TNE.

Em um estudo realizado por Agudelo, et al., (2011), com 419 pacientes em UTI, com o objetivo de estimar a incidência de complicações relacionadas e terapia nutricional (TN) em pacientes críticos, a incidência de complicações mecânicas foi de $6 \%$ para deslocamento ou saída acidental da sonda. O mal posicionamento ou saída da sonda podem ser evitados se o procedimento da instalação for realizado por profissional especializado e com acompanhamento de exames radiológicos.

Segundo Ruotolo F, et al., (2014) as pausas na infusão de dietas enterais são comuns na rotina hospitalar e ocorrem por diversos motivos, previsíveis ou imprevisíveis. Contudo, deve-se evitar que tal conduta tornase rotineira na UTI, visto que a mesma pode trazer consequências negativas ao paciente, como por exemplo, déficit calórico e protéico.

Vários são os fatores que limitam o início precoce da dieta enteral na UTI, bem como sua progressão. Dentre estes podemos citar: atraso na troca da dieta enteral seja por gotejamento inadequado/ intercorrências/esquecimentos ou outros; suspensão temporária da dieta devido paciente ter sacado a sonda; pausa na dieta para procedimento cirúrgico; não reiniciar a dieta no momento programado, entre outros. Uma grande falha observada neste estudo foi a falta de conscientização da equipe quanto à importância do aporte nutricional adequado aos pacientes de UTI, sendo indispensável relatar corretamente em prontuário essas variáveis.

Quando se aplica IQTN em uma unidade e/ou setor, é importante lembrar que o cenário clínico exige que seja organizado e padronizado conforme a realidade do setor e dispor de recursos humanos, a fim de se obter bons resultados, para que as metas sejam alcançadas e a seleção de IQTN seja realista. Os IQTNs aplicados nesse estudo não descrevem diretamente a qualidade do serviço oferecido pela instituição, mas atuam para direcionar a identificação de aspectos a serem remodelados (ALVES AHR e BORGES S, 2019).

A qualidade do cuidado é um conceito que agrega diversos componentes, como se destacam a eficácia, eficiência, acessibilidade e oportunidade; onde a aplicação de medidas baseadas em evidências, dispondo 
de tecnologia avançada, profissionais de saúde treinados e comprometidos com seus protocolos bem estabelecidos e preocupados com uma gestão de qualidade em TN, resultará em melhores benefícios para o paciente, a instituição e a sociedade (WAITZBERG DL, 2010).

Como limitação do estudo, destaca-se a coleta dos dados por meio de fichas de anotações preenchidos por terceiros, falta de profissionais para a coleta de dados em dias consecutivos e o tamanho da amostra que é muito limitada podendo os dados serem superestimados.

A interpretação dos nossos achados em relação à de outros resultados descritos na literatura reforça a condição de cada UTI e fortalece a necessidade da instituição de elaborar protocolos assistenciais, que considerem a especificidade de cada serviço, sem deixar a observação crítica das recomendações das diversas sociedades à margem.

\section{CONCLUSÃO}

Os indicadores de qualidade em terapia nutricional são instrumentos efetivos para o acompanhamento da TN ofertada. Portanto, o presente estudo demonstrou, ao final do acompanhamento, que os três indicadores de qualidade em terapia nutricional enteral aplicados, estavam de acordo com as metas pré-estabelecidas. Com os dados apresentados observou-se que apesar do setor de nutrição ter como base um protocolo de administração da NE, houve diferenças entre o planejamento nutricional e sua efetiva administração para o paciente. Como ação de melhoria, sugere-se a realização de treinamentos específicos para toda a equipe das UTI's para conscientização da importância da oferta do aporte nutricional adequado aos pacientes críticos e melhor manejo das complicações relacionadas à TNE. Ressalta-se a importância de instituir uma Equipe Multidisciplinar de Terapia Nutricional (EMTN) envolvida com o cuidado aos pacientes em uso de Terapia Nutricional, a qual assegura a viabilização e o seguimento do protocolo, com consequentes benefícios.

\section{REFERÊNCIAS}

1. AGUDELO GM, et al. Incidence of nutritional support complications in critical patients: Multicenter study. Nutritíon Hospitalaria. 2011; 26(3): 537-45.

2. ALVES AHR, BORGES S. Indicadores de qualidade em terapia enteral: avaliação da assistência nutricional ao paciente hospitalizado. Brasília - DF, BRASPEN Jornal 2019; 34 (1): 77-82.

3. FREITAS TEC, et al. Avaliação dos indicadores de qualidade da terapia de nutrição enteral em um hospital universitário, Teresina - PI; BRASPEN Jornal 2018; 33 (4): 395-401.

4. FUJINO V, NOGUEIRA LABNS. Terapia nutricional enteral em pacientes graves: revisão de literatura. Arq Ciência Saúde. 2007;14(4):220-6.

5. JESUS KMG, et al. Adequação de energia e proteína para pacientes críticos em terapia nutricional enteral. Natal RN. BRASPEN Jornal 2019; 34 (3): 293-8.

6. JOSÉ IB, et al. Target, prescription and infusion of enteral nutritional therapy of critical patients in intensive care unit, Campinas - SP. Arq Gastroenterol - 2018. v. 55 no 3 jul/set.

7. LUZ ERL, MEZZOMO TR. Nutritional status and quality indicators for enteral nutritional therapy in institutionalized patients with cerebral palsy. Demetra. 2015;10(1):189-202.

8. MARTINS JR. Fatores determinantes na inadequação entre prescrição e recebimento de terapia nutricional enteral em pacientes hospitalizado [Dissertação de mestrado]. São Paulo: Universidade de São Paulo, Faculdade de Medicina; 2012.

9. MCCLAVE SA, et al. A.S.P.E.N. Board of Directors; American College of Critical Care Medicine; Society of Critical Care Medicine. Guidelines for the Provision and Assessment of Nutrition Support Therapy in the Adult Critically III Patient: Society of Critical Care Medicine (SCCM) and American Society for Parenteral and Enteral Nutrition (A.S.P.E.N.). JPEN J Parenter Enteral Nutr. 2009;33(3):277-316.

10. MENDONÇA MR e GUEDES G. Terapia nutricional enteral em uma unidade de terapia intensiva: prescrição versus infusão. BRASPEN J. 2018; 33 (1):54-7.

11. OLIVEIRA CCG e SEGADILHA NAL. Adequação calórico proteica da terapia nutricional enteral em pacientes adultos internados em uma unidade de terapia intensiva, Rio de Janeiro, RJ, BRASPEN Jornal 2019; 34 (3): 233-8.

12. OLIVEIRA SO, et al. Impacto da adequação da oferta energética sobre a mortalidade em pacientes de UTI recebendo nutrição enteral, São Paulo - SP, Revista Brasileira Terapia Intensiva. 2011; 23(2):183-189.

13. PIOVACARI SMF, et al. Equipe Multiprofissional de Terapia Intensiva. 1 edição, Rio de Janeiro: Atheneu, 2017. p. 93.

14. RIBEIRO LMK, et al. Adequação dos balanços energético e proteico na nutrição por via enteral em terapia intensiva: quais são os fatores limitantes? Rev Bras Ter Intensiva. 2014;26(2):155-62. 
15. RIBEIRO LMK, et al. Adequação dos balanços energéticos e proteico na nutrição por via enteral em terapia intensiva: quais são os fatores limitantes?, São Paulo - SP, Rev Bras Ter Intensiva. 2014;26(2):155-162.

16. RUOTOLO F, et al. Monitoramento da adequação calórico-proteica da terapia nutricional enteral exclusiva em pacientes internados em hospital privado da cidade de São Paulo, São Paulo, SP, Revista Brasileira Nutrição Clinica 2014; 29 (3): 221-5.

17. SÁ JSM e MARSHALL NG. Indicadores de Qualidade em Terapia Nutricional como ferramenta de monitoramento da assistência nutricional no paciente cirúrgico. Revista Brasileira Nutrição Clinica 2015; 30 (2): 100-5.

18. SILVA RR, et al. Prevalência de readmissões após alta em uma Unidade de Terapia Intensiva de um hospital do interior de Rondônia. Revista Eletrônica Acervo Saúde, 2020 (42), e2871.

19. SOUZA MA e MEZZOMO TR. Estado nutricional e indicadores de qualidade em terapia nutricional de idosos sépticos internados em uma UTI. Revista Brasileira Nutrição Clinica 2016; 31 (1): 23-8.

20. TOLEDO DO e CASTRO MG. Terapia Nutricional em UTI / organização Diogo Oliveira Toledo, Melina Gouveia Castro; colaboração Alessandro Laviano ... [et al.]. - 2. Ed. - Rio de Janeiro: Rubio, 2019. 400p.; $24 \mathrm{~cm}$.

21. TOLEDO DO e CASTRO MG. Terapia Nutricional em UTI / organização Diogo Oliveira Toledo, Melina Gouveia Castro. - 1. Ed. - Rio de Janeiro: Rubio, 2015. 424p.

22. VEROTTI CCG, et al. The top ten quality indicator for nutritional therapy. Nutrition Clinica Pract. 2012;27:261-7.

23. WAITZBERG DL. Indicadores de qualidade em terapia nutricional: aplicação e resultados. São Paulo: ILSI Brasil, 2010.

24. WAITZBERG DL. Indicadores de qualidade em terapia nutricional: 10 anos de IQTN no Brasil: resultados, desafios e propostas. São Paulo: ILSI Brasil, 2018.

25. WALCZEWSKI MRM, et al. Fatores que impedem a adequação da oferta nutricional enteral em pacientes críticos. Tubarão - SC: BRASPEN JORNAL 2019; 34 (4): 329-35.

26. WEIJS PJM, et al. Optimal protein and energy nutrition decreases mortality in mechanically ventilated, critically ill patients: a prospective observational cohort study. JPEN J Parenter Enteral Nutr. 2012;36:60-8. 